\title{
Spin-Hall Voltage over a Large Length Scale in Bulk Germanium
}

\author{
F. Bottegoni, ${ }^{1, *}$ C. Zucchetti, ${ }^{1}$ S. Dal Conte, ${ }^{2}$ J. Frigerio, ${ }^{1}$ E. Carpene, ${ }^{3}$ C. Vergnaud, ${ }^{4,5}$ \\ M. Jamet, ${ }^{4,5}$ G. Isella, ${ }^{1}$ F. Ciccacci, ${ }^{1}$ G. Cerullo, ${ }^{3}$ and M. Finazzi ${ }^{1}$ \\ ${ }^{1}$ LNESS-Dipartimento di Fisica, Politecnico di Milano, Piazza Leonardo da Vinci 32, 20133 Milano, Italy \\ ${ }^{2}$ Dipartimento di Fisica, Politecnico di Milano, Piazza Leonardo da Vinci 32, 20133 Milano, Italy \\ ${ }^{3}$ IFN-CNR, Politecnico di Milano, Piazza Leonardo da Vinci 32, 20133 Milano, Italy \\ ${ }^{4}$ Université Grenoble Alpes, INAC-SPINTEC, F38000 Grenoble, France \\ ${ }^{5}$ CEA-INAC-SPINTEC, F38054 Grenoble, France
}

(Received 5 December 2016; revised manuscript received 21 February 2017; published 21 April 2017)

\begin{abstract}
We exploit the spin-Hall effect to generate a uniform pure spin current in an epitaxial $n$-doped Ge channel, and we detect the electrically induced spin accumulation, transverse to the injected charge current density, with polar magneto-optical Kerr microscopy at a low temperature. We show that a large spin density up to $400 \mu \mathrm{m}^{-3}$ can be achieved at the edges of the $100-\mu \mathrm{m}$-wide Ge channel for an applied electric field lower than $5 \mathrm{mV} / \mu \mathrm{m}$. We find that the spin density linearly decreases toward the center of the Ge bar, due to the large spin diffusion length, and such a decay is much slower than the exponential one observed in III-V semiconductors, allowing very large spin accumulations over a length scale of tens of micrometers. This lays the foundation for multiterminal spintronic devices, where different spin voltages can be exploited as inputs for magnetologic gates on the same Ge platform.
\end{abstract}

DOI: 10.1103/PhysRevLett.118.167402

Spintronics aims at electrically manipulating the carriers' spin degree of freedom in condensed-matter systems [1]. Bulk germanium is one of the most appealing materials to realize this goal, thanks to its compatibility with the $\mathrm{Si}$ platform, the long electron spin lifetime [2], and the optical properties matching the conventional telecommunication window [3]. Ideally, the design of electrically driven spintronic devices would require the generation of pure spin currents and possibly large spin voltages inside the semiconductor, avoiding external building blocks based on electrical or optical spin injection.

To this purpose, the generation of pure spin currents in the absence of magnetic and collinear electric fields is of crucial importance, since it allows achieving a spin splitting in the electrochemical potential, i.e., a spin voltage, while the carriers' electrical potential remains constant. Pure spin currents in materials can be generated through the spin-Hall effect (SHE) [4-9], which leads to the creation of a transverse spin current upon the electrical injection of a charge current as a consequence of spin-dependent scattering due to spin-orbit (SO) coupling. Since spin-to-charge interconversion effects are ubiquitous in solid-state physics, the SHE plays a fundamental role in both metals and semiconductors [7]. Indeed, since its first electrical detection [10], the SHE has turned out to be an important tool to efficiently manipulate the magnetization through spintransfer torque $[11,12]$ in ferromagnetic metals.

In doped III-V semiconductors, the charge-to-spin current conversion efficiency, also referred to as the spin-Hall angle, has been ascribed to the spin-dependent scattering of flowing electrons by ionized doping impurities [5,7-9]. Although this process results in a relatively high value of the spin-Hall angle $[5,6,13]$, in these materials spin relaxation rates are also high, limiting the spin diffusion length to about one micrometer [5,7-9,13-15] and thus hindering the achievement of sizable spin accumulation and spin voltages over micrometer length scales even at a low temperature.

In this context, germanium plays a key role thanks to its compatibility with the $\mathrm{Si}$ platform, its large spin-orbit interaction, and the long-predicted $[16,17]$ and experimentally measured [18,19] electron spin lifetime. All these features make $\mathrm{Ge}$ a promising candidate for the implementation of new spin functionalities on $\mathrm{Si}$. Spin accumulation in the conduction band of bulk Ge can be obtained by electrical spin injection [19-21] or optical spin orientation [22-24]. It can be then detected by the inverse spin-Hall effect, which can take place directly in Ge [25] or in a thin metal layer deposited on Ge with a large SO interaction $[22,24]$. In this respect, spin injection or detection schemes using ferromagnetic metals generally introduce parasitic interfacial effects [26] related to the metal-semiconductor junction [27] or interface states [28], which strongly affect the spin transport properties of the semiconductor. Here, to avoid such parasitic effects, we directly probe the spin transport and dynamics in bulk Ge using magneto-optical Kerr microscopy [5] to measure the spin density induced across an electrically biased Ge channel by the SHE.

We have fabricated a $250 \times 100 \mu \mathrm{m}^{2}$-large and 3- $\mu \mathrm{m}$ thick $n$-doped Ge channel deposited on a $500-\mu$ m-thick high-resistivity $\mathrm{Si}(001)$ substrate (see Supplemental Sec. A for further details [29]). The Ge layer is $n$-doped with a phosphorus concentration $N=2.5 \times 10^{18} \mathrm{~cm}^{-3}$, and its resistivity is $\rho=1.05 \times 10^{-4} \Omega \mathrm{m}$ at $T=20 \mathrm{~K}$. The $2 \mathrm{D}$ 


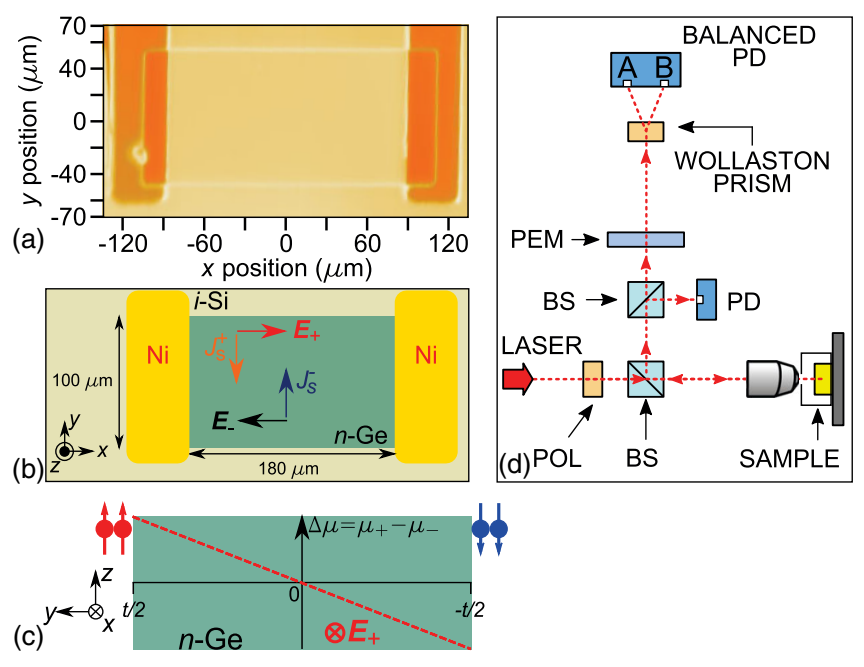

FIG. 1. (a) $2 \mathrm{D}$ optical reflectivity map at $T=20 \mathrm{~K}$ of the $n$-doped Ge channel, grown on a high-resistivity $\mathrm{Si}(001)$ substrate. (b) Geometry of the SHE: A constant positive (negative) electric field $E_{+(-)}$is applied along the $x$ axis between the two Ni Ohmic contacts of the Ge channel, resulting in a negative (positive) pure spin current $\mathbf{J}_{\mathrm{s}}$ along the $y$ axis. (c) Scheme of the spin electrochemical potential along the Ge channel width ( $y$ axis) given by the SHE. (d) Sketch of the magneto-optical Kerr microscopy setup. BS, beam splitter; PEM, photoelastic modulator; PD, photodiode.

optical reflectivity map of the sample is shown in Fig. 1(a). A dc bias is applied in Ge along the $x$ axis between two 100-nm-thick Ni pads, acting as Ohmic contacts at the edges of the Ge channel [see Fig. 1(b)]. The injection of a charge current density $\mathbf{J}_{c}$ generates a pure spin current density $\mathbf{J}_{s}=\gamma_{\mathrm{SH}} \mathbf{J}_{c} \times \mathbf{u}_{P}$ due to the SHE $[4,8,9]$, where $\gamma_{\mathrm{SH}}$ is the spin-Hall angle and $\mathbf{u}_{P}$ is the unit vector parallel to the spin polarization vector. Since we exploit a polar Kerr configuration, i.e., the propagation direction of the incident light is perpendicular to the semiconductor channel surface, we are sensitive only to the out-of-plane direction of the spin polarization along the $z$ axis. Then, applying a positive (negative) electric field $E_{+(-)}$parallel to the $x$ axis [see Fig. 1(b)], a negative (positive) $\mathbf{J}_{s}$ is generated along the $y$ axis, yielding spin accumulations of opposite sign at the edges of the Ge channel. This is illustrated in Fig. 1(c) by the spin electrochemical potential profile along the $\mathrm{Ge}$ channel width. The Kerr microscopy setup is sketched in Fig. 1(d). The linearly polarized light from a continuouswave laser diode at $1.55 \mu \mathrm{m}$ wavelength $(h \nu=0.8 \mathrm{eV})$ enters a linear polarizer, and it is then focused onto the sample surface inside the cryostat at $T=20 \mathrm{~K}$ with the use of an objective with 0.7 numerical aperture, giving a full width at half maximum focal spot size of $w \approx 1.5 \mu \mathrm{m}$. The reflected beam is collected by a polarization-maintaining beam splitter. A second beam splitter separates the light beam along two optical arms: The first one is devoted to the detection of the reflectivity $R$ with the use of a InGaAs photodetector, and the second one records the Kerr ellipticity signal $\vartheta_{\text {ell }}$ with a balanced photodiode bridge, modulated at $50 \mathrm{kHz}$ by a photoelastic modulator. The demodulated voltage signal is detected with a lock-in amplifier.

Note that in bulk Ge the electrical conduction occurs at the indirect gap ( $L$ valleys), lying $0.74 \mathrm{eV}$ above the valence band maximum at a low temperature. The incident photon energy $h \nu=0.8 \mathrm{eV}$, quasiresonant with respect to the Ge direct band gap $E_{d}$ at the $\Gamma$ point $\left(E_{d}=0.88 \mathrm{eV}\right.$ at $T=20 \mathrm{~K}$ ), is chosen to enhance the magnitude of the Kerr signal while avoiding optical absorption at $\Gamma$ [38].

Figure 2(a) shows the profiles for the optical reflectivity $R$ (top panel) and Kerr ellipticity $\vartheta_{\text {ell }}$ (bottom panel) measured at $T=20 \mathrm{~K}$ across the $\mathrm{Ge}$ channel, corresponding to a scanned area of $40 \times 130 \mu \mathrm{m}^{2}$ around the center of the sample and averaged along the $x$ direction [see Fig. 1(a)]. The incident optical power is $W=2 \mathrm{~mW}$. A positive (red dots) and negative (black dots) bias of $840 \mathrm{mV}$ resulting in an electric field $E_{+(-)}=4.7 \mathrm{mV} / \mu \mathrm{m}$ is applied along $+x\left(E_{+}\right)$ and $-x\left(E_{-}\right) . \vartheta_{\text {ell }}$ is reported only between $y=-42$ and $42 \mu \mathrm{m}$, which corresponds to the scanned region where $R$ is flat, to avoid possible optical artifacts related to the $\mathrm{Ge}$ channel edges. The reversal of the electric field does not change, as expected, the optical reflectivity profile, whereas $\vartheta_{\text {ell }}$ shows a quasilinear behavior across the Ge channel with a slope changing sign when the applied electric field is reversed. Since $\vartheta_{\text {ell }}$ is proportional to the spin density $n_{s}$, the observation of the Kerr ellipticity profile in Fig. 2(a) is indicative of a spin accumulation, with an absolute value increasing from the center to the edges of the $\mathrm{Ge}$ channel along the $y$ axis, reaching the maximum value $\vartheta_{\text {ell }}=70 \pm 40 \mu \mathrm{rad}$. The difference between the two averaged spin accumulation profiles, obtained with opposite electric fields, is presented in Fig. 2(b), which highlights the linear character of $\vartheta_{\text {ell }}$ with respect to the position $y$ within the experimental error, whereas a complete 2D map of $\vartheta_{\text {ell }}$ is shown in Fig. 3.

By solving the spin drift-diffusion equations for a charge current density $\mathbf{J}_{c}$ and the electrically induced spin current density $\mathbf{J}_{s}$, flowing along the $x$ and $y$ axis, respectively, we derive the spin density profile $[39,40]$

$$
n_{s}(y)=\frac{L_{s} \sigma_{\mathrm{SH}}}{e D_{\mathrm{el}}} \operatorname{sech}\left(\frac{t}{2 L_{s}}\right) \sinh \left(\frac{y}{L_{s}}\right) E
$$

where $\sigma_{\mathrm{SH}}=\gamma_{\mathrm{SH}} \sigma_{\mathrm{Ge}}$ is the spin-Hall conductivity, $\sigma_{\mathrm{Ge}}=$ $9.5 \times 10^{3}(\Omega \mathrm{m})^{-1}$ being the electrical conductivity of the Ge channel, $e$ the electron charge, $t$ the width of the Ge channel $\left(100 \mu \mathrm{m}\right.$ for our sample), $D_{\mathrm{el}}=23 \mathrm{~cm}^{2} / \mathrm{s}$ the electron diffusion coefficient, and $L_{s}$ the spin diffusion length. When $L_{s} \approx t$, one can approximate $n_{s}(y) \propto\left(y / L_{s}\right)$, which reproduces the linear spatial dependence experimentally observed in Fig. 2(b). This indicates that $L_{s}$ in bulk Ge at a low temperature is large and comparable with the width of our sample, in agreement with theoretical calculations of 

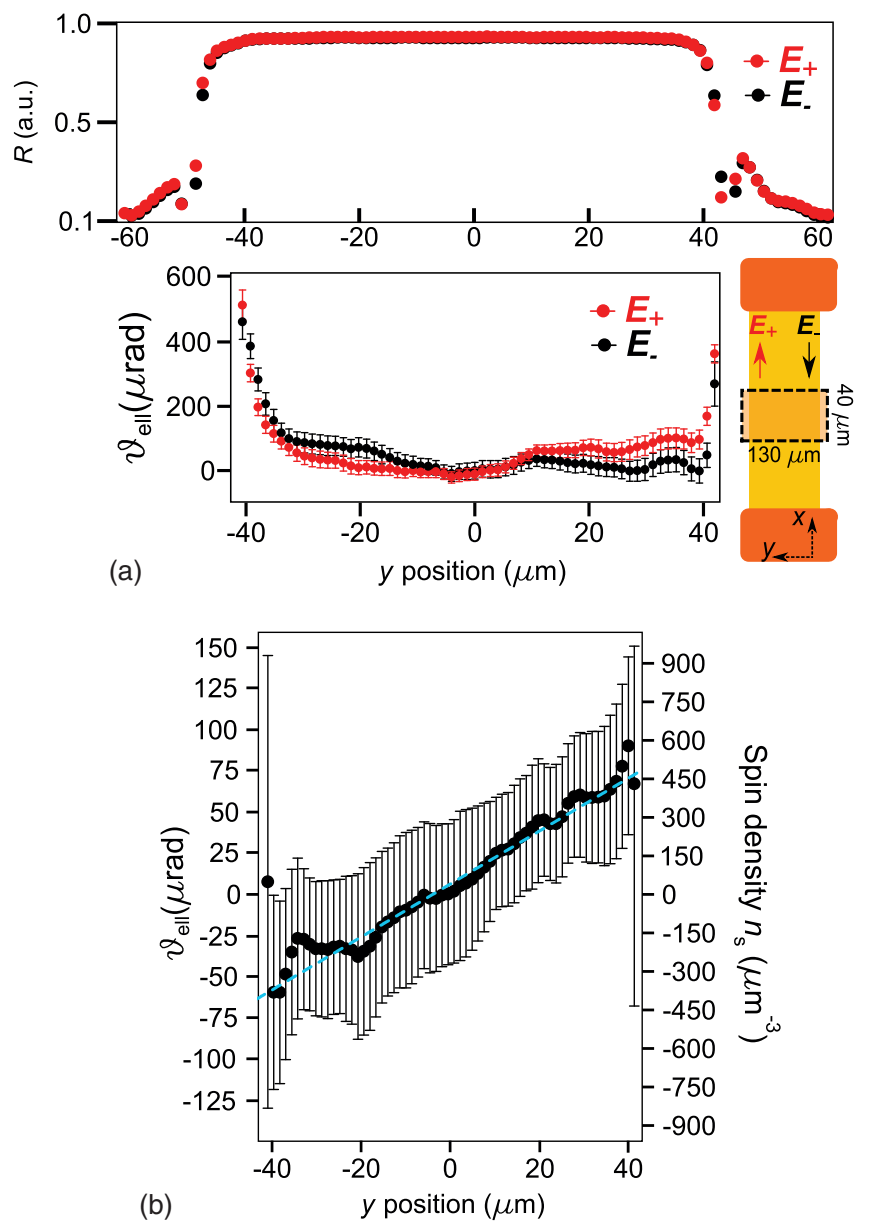

FIG. 2. (a) Average of 40 line profiles of the optical reflectivity $R$ (top panel) and the Kerr ellipticity $\vartheta_{\text {ell }}$ (bottom panel) across the Ge channel for an applied positive $E_{+}$(red dots) and negative $E_{(-)}$(black dots) uniform electric field $E_{+(-)}=4.7 \mathrm{mV} / \mu \mathrm{m}$. The scanned area is $40 \times 130 \mu \mathrm{m}^{2} . \vartheta_{\text {ell }}$ is shown in the range between $y=-42$ and $42 \mu \mathrm{m}$. (b) Difference of the Kerr ellipticity profiles in (a) (left $y$ axis) and electrically induced spin density $n_{s}$ across the Ge channel (right $y$ axis), as obtained from the calibration procedure described in Supplemental Material, Sec. C [29]. The dashed light blue line is a linear fit of the experimental data from Eq. (1).

a long electron spin lifetime at the $L$ valleys in Ge at a low temperature [16]. This explains the dramatic difference between the experimental data in Fig. 2(b) and those reported in Ref. [5], where, in a similar experimental geometry, a non-null magneto-optical Kerr signal is detected only at the edges of a GaAs channel, due to the lower value of $L_{s}$. In our case, it is not possible to experimentally give a precise value of $L_{s}$ but only a lower bound estimation, since any value $L_{s} \approx t$ would result in a linear behavior of $n_{s}$.

To confirm this hypothesis, we estimate the electron spin lifetime $\tau_{s}$ by accounting for the spin-flip rates related to inter- and intravalley scattering [16] $\left(1 / \tau_{s \text {-inter }}\right.$ and $1 / \tau_{s \text {-intra }}$, respectively) at the $L$ point of the Ge Brillouin zone and the

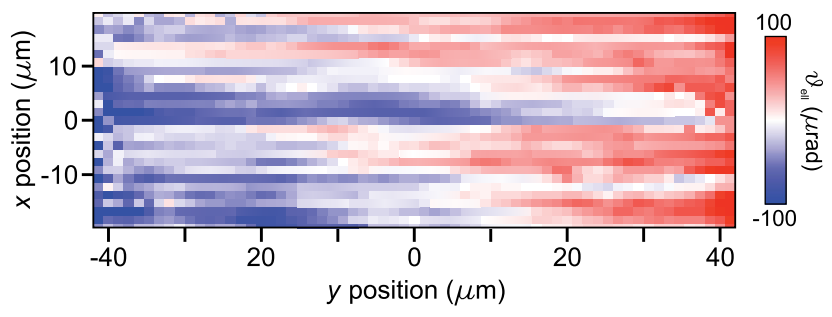

FIG. 3. Kerr ellipticity map across the Ge channel, obtained as the difference between measurements with opposite applied electric field $E_{+(-)}=4.7 \mathrm{mV} / \mu \mathrm{m}$. The scanned area is $40 \times$ $84 \mu \mathrm{m}^{2}$ around the center of the sample, corresponding to a region where the reflectivity $R$ is flat. Measurements have been performed at $T=20 \mathrm{~K}$ with an incident power of $W=2 \mathrm{~mW}$.

ionized-impurity scattering rate $1 / \tau_{s \text {-imp }}$ (see Supplemental Sec. B for further details [29]): $1 / \tau_{s}=1 / \tau_{s \text {-inter }}+$ $1 / \tau_{s \text {-intra }}+1 / \tau_{s \text {-imp. }}$ Since, at $T=20 \mathrm{~K}, \quad \tau_{s \text {-inter }}$, $\tau_{s \text {-intra }} \gg \tau_{s \text {-imp }}$, we obtain $\tau_{s} \approx \tau_{s \text {-imp }}=420 \mathrm{~ns}$, and, introducing the electron diffusion coefficient $D_{\text {el }}$, we estimate $L_{s}=\sqrt{D_{\mathrm{el}} \tau_{s}}=31 \mu \mathrm{m}$. It is worth noticing that in the calculation of $\tau_{s \text {-imp }}$ only the short-range interaction with impurities, driven by SO coupling, has been taken into account, since the long-range (Coulomb) interaction is generally negligible in multivalley semiconductors [41]. On the other hand, the long-range interaction should not vary by doping Ge with different impurities (As, Sb, and P), so that further SHE experiments with As or Sb-doped Ge could allow addressing the role of the different interaction potentials.

A quantitative analysis of the Kerr ellipticity profiles can be performed by exploiting the optical spin orientation technique to inject spin-polarized electrons in Ge $[2,9,22]$. Indeed, dipole selection rules for optical transitions with circularly polarized light allow generating a spin-oriented electron population in the conduction band of Ge. In this case, one can estimate the spin-polarized electron density $n^{\text {opt }}$ injected into the semiconductor (see Supplemental Sec. C for further details [29]), which can be directly related to the Kerr ellipticity $\vartheta_{\text {ell }}^{\text {opt }}$ measured under optical spin injection conditions.

From this calibration procedure, we can then calculate the electrically induced spin density $n_{s}$ in the Ge channel, generated by the spin-Hall effect, for the data set in Fig. 2(b). We find $n_{s} \approx \pm 400 \mu \mathrm{m}^{-3}$ for $y= \pm 42 \mu \mathrm{m}$, indicative of a large spin accumulation in correspondence to the edges of the Ge channel [see Fig. 2(b)]. Such a value is approximatively 2 orders of magnitude larger than that reported for an InGaAs channel with a similar size and electrical resistivity [5], whereas it is comparable to the ones measured in Ref. [13] for GaAs channels with different doping densities.

However, at variance with III-V semiconductors, the spin density linearly decreases toward the center of the Ge bar, thanks to the large value of $L_{s}$, allowing for 


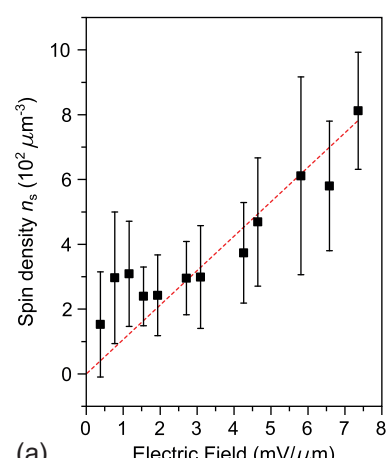

(a)
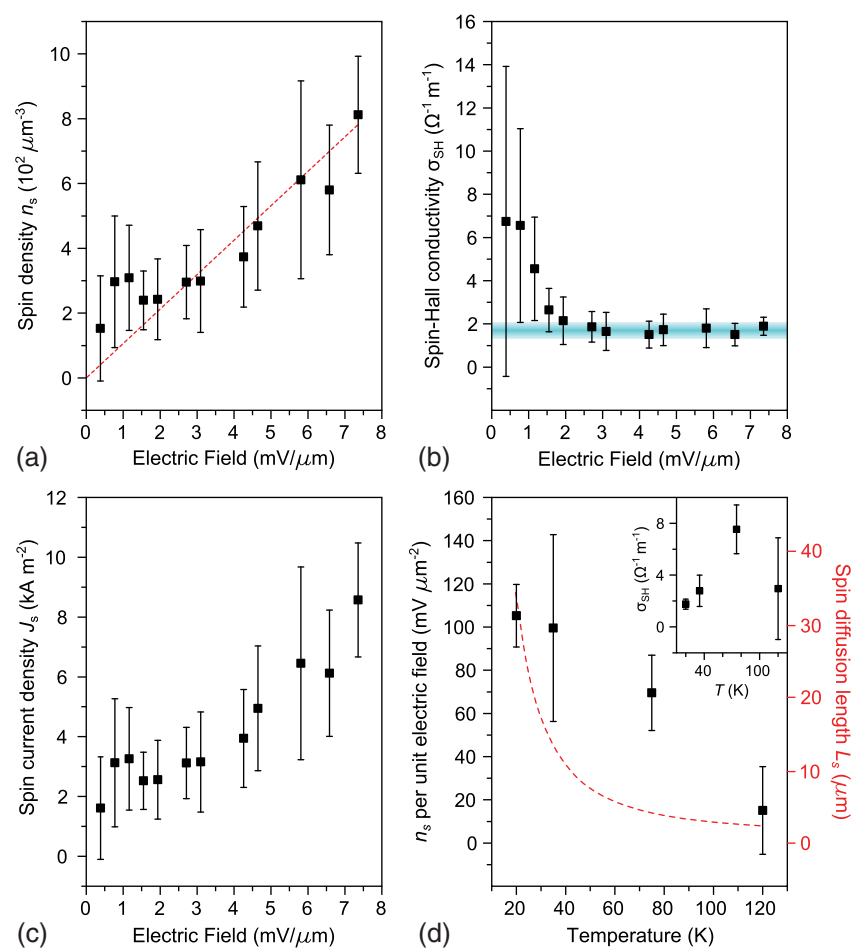

FIG. 4. (a) Electrically induced spin density $n_{s}$ across the Ge channel width as a function of the applied electric field. The dashed red line represents a linear fit of the experimental data from Eq. (1). (b) Spin-Hall conductivity $\sigma_{\mathrm{SH}}$ of the Ge channel as a function of the applied electric field at $T=20 \mathrm{~K}$. The light blue area identifies the averaged value of $\sigma_{\mathrm{SH}}$, weighed with the experimental error. (c) Spin current density $J_{s}$ for $y=0$ at $T=20 \mathrm{~K}$, estimated through the spin drift-diffusion model of Eq. (1) from the experimental data set in (a). (d) Electrically induced spin density $n_{s}$ for a unit electric field and $y=42 \mu \mathrm{m}$ as a function of the temperature. The red dashed line corresponds to the estimated temperature dependence of the spin diffusion length $L_{s}$ (see Supplemental Sec. B [29]). Inset: Spin-Hall conductivity $\sigma_{\mathrm{SH}}$ of the Ge channel as a function of the temperature.

the exploitation of sizable spin accumulations over tens of micrometers. Indeed, the combination of large spin voltages and large length scales, which is simply inaccessible to GaAs-based semiconductors, is suitable for the design of multiterminal spintronic devices, where different spin voltages can be used as inputs for multiple magnetologic gates on the same Ge platform [42].

According to Eq. (1), a linear fit of the experimental data in Fig. 2(b) for $t=100 \mu \mathrm{m}, E=4.7 \mathrm{mV} / \mu \mathrm{m}$, and $L_{s}=$ $31 \mu \mathrm{m}$ yields $\sigma_{\mathrm{SH}}=2 \pm 1(\Omega \mathrm{m})^{-1}$, which corresponds to a spin-Hall angle $\gamma_{\mathrm{SH}}=(2 \pm 1) \times 10^{-4}$, in line with previously reported values in $n$-doped bulk Ge [25].

Figure 4(a) shows the dependence of $n_{s}$, measured at $y=42 \mu \mathrm{m}$, on the applied electric field $E$ with an incident photon energy $h \nu=0.8 \mathrm{eV}$ and an optical power $W=2 \mathrm{~mW}$. The spin accumulation profile for every value of the applied electric field $E$ is linearly fitted by Eq. (1), using once again $t=100 \mu \mathrm{m}, y=42 \mu \mathrm{m}$, and $L_{s}=31 \mu \mathrm{m}$. As shown in Fig. 4(b), $\sigma_{\mathrm{SH}}$ remains nearly constant through all the investigated bias range, and its averaged value corresponds to $\sigma_{\mathrm{SH}}=1.8 \pm$ $0.2(\Omega \mathrm{m})^{-1}$. The spin drift-diffusion equations of Refs. $[39,40]$ can be exploited to obtain the spin current density profile $J_{s}(y)=-D_{\mathrm{el}} \partial_{y} n_{s}-\sigma_{\mathrm{SH}} E$ with the proper boundary conditions $J_{s}(y= \pm t / 2)=0$. In this case, the spin current density $J_{s}$ at the center of the Ge channel is then calculated using $y=0, t=100 \mu \mathrm{m}$, and $\sigma_{\mathrm{SH}}=1.8 \pm$ $0.2(\Omega \mathrm{m})^{-1}$ [see Fig. 4(c)], which reproduces the linear behavior of $n_{s}$ as a function of $E$.

Finally, the electrically induced spin density $n_{s}$ per unit electric field as a function of the temperature is shown in Fig. 4(d). For each temperature, the dependence of the spin accumulation on the electric field has been measured and the slopes of the different curves together with the corresponding experimental error are plotted, suggesting a decrease of $n_{s}$ with $T$. We attribute this behavior to the temperature dependence of the electron spin lifetime $\tau_{s}$, which decreases as spin relaxation phenomena become more efficient with increasing temperature. By explicitly considering the temperature dependence of $L_{s}$ that can be estimated from the spin-flip rates mentioned before (see Supplemental Sec. B for further details [29]), we exploit Eq. (1) to estimate the temperature dependence of $\sigma_{\mathrm{SH}}$ [see the inset in Fig. 4(d)]. We find that, within the experimental error, $\sigma_{\mathrm{SH}}$ and-consequently- $\gamma_{\mathrm{SH}}$ are almost constant, indicating that the charge-to-spin conversion efficiency in bulk $\mathrm{Ge}$ is not degraded at least up to $120 \mathrm{~K}$.

In conclusion, we have directly probed the electrically induced spin accumulation across the Ge channel using the SHE and magneto-optical Kerr microscopy. Our experimental results give direct access to the groundwork of spin dynamics in bulk Ge and highlight the great potential of this material in the design of integrated electrically driven spintronic devices. Indeed, $L_{s}$ values in the range of tens of micrometers allow for a reliable transport of spin over large distances, paving the way for the design of complementary Si-Ge spin interconnects [43]. The favorable conduction band-edge alignment between $\mathrm{Si}$ and $\mathrm{Ge}$ heterostructures should make this kind of spin architecture easier, since it is possible to generate a large spin accumulation in $\mathrm{Ge}$ through the SHE and then transfer it in $\mathrm{Si}$, thus overcoming early challenges for spin injection and detection in this material [44]. Moreover, Ge(111)-based nanostructures are promising platforms to probe the SHE, since the straininduced removal of the valley degeneracy at the $L$ point should drastically increase the spin diffusion length [45]. Finally, the generation of very high spin voltages and currents over tens of micrometers in Ge paves the way for spin manipulation in low-dimensional Ge heterostructures, where electrical control of spin-orbit interaction can also be implemented.

Partial funding is acknowledged to the CARIPLO foundation through SEARCH-IV (2016-0623), to 
European Union Horizon 2020 Program under Grant Agreement No. 696656 Graphene Core 1, and to the French National Research Agency through the ANR project SiGeSPIN No. ANR-13-BS10-0002.

*federico.bottegoni@polimi.it

[1] I. Zutić, J. Fabian, and S. Das Sarma, Rev. Mod. Phys. 76, 323 (2004).

[2] F. Pezzoli, F. Bottegoni, D. Trivedi, F. Ciccacci, A. Giorgioni, P. Li, S. Cecchi, E. Grilli, Y. Song, M. Guzzi, H. Dery, and G. Isella, Phys. Rev. Lett. 108, 156603 (2012).

[3] M. Hochberg and T. Baehr-Jones, Nat. Photonics 4, 492 (2010).

[4] J. E. Hirsch, Phys. Rev. Lett. 83, 1834 (1999).

[5] Y. K. Kato, R. C. Myers, A. C. Gossard, and D. D. Awschalom, Science 306, 1910 (2004).

[6] N. P. Stern, S. Ghosh, G. Xiang, M. Zhu, N. Samarth, and D. D. Awschalom, Phys. Rev. Lett. 97, 126603 (2006).

[7] T. Jungwirth, J. Wunderlich, and K. Olejník, Nat. Mater. 11, 382 (2012).

[8] M. I. D’yakonov and V. I. Perel, JETP Lett. 13, 467 (1971).

[9] M. I. Dyakonov and A. V. Khaetskii, in Spin Physics in Semiconductors, edited by M. I. Dyakonov, Springer Series in Solid-State Sciences Vol. 157 (Springer, Berlin, 2008).

[10] S. O. Valenzuela and M. Tinkham, Nature (London) 442, 176 (2006).

[11] K. Ando, S. Takahashi, K. Harii, K. Sasage, J. Ieda, S. Maekawa, and E. Saitoh, Phys. Rev. Lett. 101, 036601 (2008).

[12] I. M. Miron, K. Garello, G. Gaudin, P.-J. Zermatten, M. V. Costache, S. Auffret, S. Bandiera, B. Rodmacq, A. Schuhl, and P. Gambardella, Nature (London) 476, 189 (2011).

[13] S. Matsuzaka, Y. Ohno, and H. Ohno, Phys. Rev. B 80, 241305 (2009).

[14] J. M. Kikkawa and D. D. Awschalom, Phys. Rev. Lett. 80, 4313 (1998).

[15] F. Bottegoni, H.-J. Drouhin, G. Fishman, and J.-E. Wegrowe, Phys. Rev. B 85, 235313 (2012).

[16] P. Li, Y. Song, and H. Dery, Phys. Rev. B 86, 085202 (2012).

[17] P. Li, J. Li, L. Qing, H. Dery, and I. Appelbaum, Phys. Rev. Lett. 111, 257204 (2013).

[18] C. Guite and V. Venkataraman, Appl. Phys. Lett. 101, 252404 (2012).

[19] Y. Zhou, W. Han, L.-T. Chang, F. Xiu, M. Wang, M. Oehme, I. A. Fischer, J. Schulze, R. K. Kawakami, and K. L. Wang, Phys. Rev. B 84, 125323 (2011).

[20] A. Jain et al., Phys. Rev. Lett. 109, 106603 (2012).

[21] S. Dushenko, M. Koike, Y. Ando, T. Shinjo, M. Myronov, and M. Shiraishi, Phys. Rev. Lett. 114, 196602 (2015).

[22] F. Bottegoni, M. Celebrano, M. Bollani, P. Biagioni, G. Isella, F. Ciccacci, and M. Finazzi, Nat. Mater. 13, 790 (2014).
[23] E. J. Loren, B. A. Ruzicka, L. K. Werake, H. Zhao, H. M. van Driel, and A. L. Smirl, Appl. Phys. Lett. 95, 092107 (2009).

[24] F. Bottegoni, A. Ferrari, S. Cecchi, M. Finazzi, F. Ciccacci, and G. Isella, Appl. Phys. Lett. 102, 152411 (2013).

[25] J.-C. Rojas-Sánchez, M. Cubukcu, A. Jain, C. Vergnaud, C. Portemont, C. Ducruet, A. Barski, A. Marty, L. Vila, J.-P. Attané, E. Augendre, G. Desfonds, S. Gambarelli, H. Jaffrès, J.-M. George, and M. Jamet, Phys. Rev. B 88, 064403 (2013).

[26] O. Txoperena, Y. Song, L. Qing, M. Gobbi, L. E. Hueso, H. Dery, and F. Casanova, Phys. Rev. Lett. 113, 146601 (2014).

[27] S. Sharma, A. Spiesser, S. P. Dash, S. Iba, S. Watanabe, B. J. van Wees, H. Saito, S. Yuasa, and R. Jansen, Phys. Rev. B 89, 075301 (2014).

[28] M. Tran, H. Jaffrès, C. Deranlot, J.-M. George, A. Fert, A. Miard, and A. Lemaître, Phys. Rev. Lett. 102, 036601 (2009).

[29] See Supplemental Material at http://link.aps.org/ supplemental/10.1103/PhysRevLett.118.167402 for the characterization of the sample, the calculation of the spin diffusion length as a function of the temperature and the calibration of the optical setup, which includes Refs. [30-37].

[30] P. P. Edwards and M. J. Sienko, Phys. Rev. B 17, 2575 (1978).

[31] M. N. Alexander and D. F. Holcomb, Rev. Mod. Phys. 40, 815 (1968).

[32] P. P. Debye and E. M. Conwell, Phys. Rev. 93, 693 (1954).

[33] S. S. Li and F. Lindholm, Proc. IEEE 56, 1256 (1968).

[34] Fundamental of Carrier Transport, 2nd ed., edited by M. Lundstrom (Cambridge University Press, Cambridge, United Kingdom, 2000).

[35] Spin Physics in Semiconductors Springer Series in SolidState Sciences Vol. 187, edited by M. I. Dyakonov and A. V. Khaetskii (Springer, Berlin, 2008).

[36] F. Bottegoni, A. Ferrari, G. Isella, M. Finazzi, and F. Ciccacci, Phys. Rev. B 88, 121201 (2013).

[37] G. G. Macfarlane, T. P. McLean, J. E. Quarrington, and V. Roberts, Phys. Rev. 108, 1377 (1957).

[38] D. Mitchell and R. Wallis, Phys. Rev. 131, 1965 (1963).

[39] I. Žutić, J. Fabian, and S. Das Sarma, Phys. Rev. B 64, 121201 (2001).

[40] W.-K. Tse, J. Fabian, I. Žutić, and S. Das Sarma, Phys. Rev. B 72, 241303 (2005).

[41] Y. Song, O. Chalaev, and H. Dery, Phys. Rev. Lett. 113, 167201 (2014).

[42] H. Dery, P. Dalal, Ł. Cywiński, and L. J. Sham, Nature (London) 447, 573 (2007).

[43] H. Dery, Y. Song, P. Li, and I. Žutić, Appl. Phys. Lett. 99, 082502 (2011).

[44] I. Žutić, J. Fabian, and S. C. Erwin, Phys. Rev. Lett. 97, 026602 (2006).

[45] E.-S. Liu, J. Nah, K. M. Varahramyan, and E. Tutuc, Nano Lett. 10, 3297 (2010). 